Proc Indian Natn Sci Acad 81 No. 1 February 2015 Special Issue, pp. 135-151

(C) Printed in India.

DOI: $10.16943 /$ ptinsa/2015/v81i1/48061

\title{
Exploring the QCD Critical Region in the QCD-Like Two Flavor Models VIVEK KUMAR TIWARI ${ }^{\star}$ \\ Department of Physics, University of Allahabad, Allahabad 211 002, India
}

(Received on 2 May 2014; Accepted on 10 August 2014)

\begin{abstract}
We have calculated the phase diagram for exploring the critical behavior around the critical end-point (CEP) and located the chiral limit existence of the tri-critical point (TCP) in the $\mu$ and T plane of the Polyakov loop extended Quark Meson Model (PQM) and the pure Quark Meson (QM) model which become effective Quantum-chromodynamics (QCD) like models due to the augmentation of the effective potential by the renormalized fermionic vacuum one loop fluctuation. These models yield the second order transition at $\mu=0$ on the temperature axis after incorporating the fermionic vacuum correction. The proximity of the TCP to the QCD critical end-point (CEP) has been quantified in the phase diagram. We have plotted the contours of appropriately normalized constant quark number susceptibility and scalar susceptibility around the CEP in different model scenarios. In order to investigate the qualitative as well as quantitative effect of the fermionic vacuum term and the Polyakov loop potential, on the critical behavior around CEP, we have compared the shape of these contours as obtained in different model calculations. Further, we have computed and compared the critical exponents resulting from the divergence of quark number susceptibility at the CEP in different model scenarios. The possible influence of the TCP on the critical behavior around CEP, has also been discussed.
\end{abstract}

\section{Key Words : Quark Gluon Plasma (QGP); Quantum Chromodynamics (QCD); Chiral Symmetry; Confinement; Critical End Point (CEP), Tricritical Point (TCP)}

\section{Introduction}

Under the extreme conditions of high temperature and/or density, the normal hadronic matter dissolves into its quark and gluon constituents. One gets a phase transition to the collective form of matter known as the Quark Gluon Plasma(QGP) (Rischke, 2004; Mueller, 1995; Svetitsky, 1986). Study of the different aspects of this phase transition, is a tough and challenging task because Quantum Chromodynamics(QCD)-

*Authorfor Correspondence : E-mail: vivekkrt@gmail.com 
the theory of strong interaction- becomes nonperturbative in the low energy limit. However the QCD vacuum reveals itself through the process of spontaneous chiral symmetry breaking and phenomenon of color confinement. Chiral condensate is the order parameter for the spontaneously broken chiral $S U_{A}\left(N_{f}\right)$ symmetry for the $N_{f}$ flavours of massless quarks in the QCD Lagrangian and one gets $\left(N_{f}^{2}-1\right)$ massless Goldstone bosons. Since the observed pions-pseudo Goldstone bosons- in nature are very light, we have an approximate chiral symmetry for the real QCD with two falvours of light $u$ and d quarks. In the opposite limit of infinitely heavy quarks, the QCD becomes a pure $S U\left(N_{c}\right)$ gauge theory having the global $Z\left(N_{c}\right)$ symmetry of the center of gauge group. This center symmetry of the hadronic vacuum, gets spontaneously broken in the high temperature/density regime of QGP. The expectation value of the Wilson line (Polyakov loop) is related to the free energy of a static color charge. It vanishes in the confining phase as the quark has infinite free energy and becomes finite in the deconfined phase. Hence the Polyakov loop serves as the order parameter of the confinement-deconfinement phase transition (Polyakov, 1978). Even though the center symmetry is always broken with the inclusion of dynamical quarks in the system, one can regard the Polyakov loop as an approximate order parameter because it is a good indicator of a rapid crossover in the confinement-deconfinement transition (Pisarski, 2000; Layek et al., 2006).

Construction and mapping of the phase diagram in the quark chemical potential and temperature plane is the prime challenge before the experimental as well as theoretical QGP community. On the temperature axis, the chiral transition at zero quark chemical potential with almost physical quark masses, has been well established to be a crossover in recent lattice QCD simulations (Aoki et al., 2006, 2009; Bazavov et al., 2012; Borsanyi et al., 2010). Effective chiral model studies (Pisarski and Wilczek, 1984) predict first order phase transition at lower temperatures on the chemical potential axis. Thus the existence of a critical end point (CEP) has been suggested in the phase diagram based on model studies (Asakawa and Yazaki, 1989; Barducci et al., 1989, 1990, 1994; Berges and Rajagopal, 1999) together with the inputs from lattice simulations (Forcrand and Philipsen, 2002; Fodor and Katz, 2002; Fodor et al., 2003; Allton et al., 2003, 2005). The first order transition line starting from the lowest temperature on the chemical potential axis, terminates at the CEP which is a genuine singularity of the QCD free energy. Here the phase transition turns second order and its criticality belongs to the three dimensional Ising universality class (Hatta and Ikeda, 2003; Fujii, 2003; Fukushima, 2001; Son and Stephanov, 2004; Aoki et al., 2006; Gupta et al., 2011). The precise location of the CEP is highly sensitive to the value of the strange quark mass. Lattice QCD predictions at non zero chemical potential are much more difficult due to the QCD action becoming complex on account of the fermion sign problem (Karsch, 2002). There is evidence for a CEP at finite $\mu$ (Forcrand and Philipsen, 2002; Fodor and Katz, 2002; Fodor et al., 2003) from a Taylor expansion of QCD pressure around $\mu=0$, however in another lattice study, finite chemical potential extrapolations provide 
some limitations and can rule out the existence of a CEP for small $\mu /$ T ratios (Philipsen, 2011). In the chiral limit of zero up and down quark masses, the chiral phase transition is of second order at zero $\mu$ and the static critical behavior is expected to fall in the universality class of the $\mathrm{O}(4)$ spin model in three dimensions (Pisarski and Wilczek, 1984). Thus the existence of CEP for real life two flavor QCD implies that two flavor massless QCD has a tricritical point(TCP) at which the second order $\mathrm{O}(4)$ line of critical points ends.

If the signals are not washed out due to the expansion of the colliding system, the critical point might be located in the phase diagram by the observation of non monotonic behavior of number fluctuations in its vicinity (Stephananov et al., 1999, 1998; Jeon and Koch, 2000; Ejiri et al., 2006). Recently "beam energy scan" program dedicated to the search of critical point has been started at the Relativistic Heavy Ion Collider (RHIC, Brookhaven National Laboratory) experiments (Adams et al., 2005; Aggarwal et al., 2005). The Compressed Baryonic Matter (CBM) experiment (GSI-Darmstadt) at the facility for Antiproton and Ion Research (FAIR) and the Nuclotron-Based Ion Collider facility (NICA) at the Joint Institute for Nuclear Research (JINR), will also be looking for the signatures of critical end point. Characteristic signatures of the conjectured CEP for experiments have been discussed in Refs. (Mohanty, 2009; Koch, 2008; Luo et al., 2012). The critical region around CEP is not point-like but has a much richer structure. This issue is being pursued actively in a variety of effective model studies (Hatta and Ikeda, 2003; Fujii, 2003; Barducci et al., 1994; Halasz et al., 1998; Harada and Shibata, 1999; Brouzakis and Tetradis, 2004; Nonaka and Asakawa, 2005; Fuji and Ohtani, 2004; Costa et al., 2009). Present investigation has been done in the framework of extended linear sigma model called the Polyakov loop Quark Meson (PQM) model (Schaefer and Wanger, 2007, 2009; Schaefer et al., 2009, 2010; Mao et al., 2010; Gupta and Tiwari, 2010; Marko and Szep, 2010; Skokov et al., 2010; Herbst et al., 2011) where the chiral order parameter and the Polyakov loop order parameter has been simultaneously coupled to the quark degrees of freedom. Skokov et al., (2010) incorporated the renormalized fermionic vacuum one loop correction-used to get neglected in the current literature under the no sea mean field approximation- in the thermodynamic potential of the Quark Meson (QM) model. Now QM model becomes an effective QCD-like model because now it can reproduce the second order chiral phase transition on the temperature axis at zero chemical potential $\mu=0$ as expected from the universality arguments (Pisarski and Wilczek, 1984). Recently, we (Gupta and Tiwari, 2012) generalized the proper accounting of renormalized fermionic vacuum fluctuation in the two flavour PQM model to the non-zero chemical potentials and found that the position of CEP shifts to a significantly higher chemical potential in the $\mu$ and $\mathrm{T}$ plane of the phase diagram, due to the influence of fermionic vacuum term in our PQMVT (PQM model with vacuum term) model calculations. The fermionic vacuum correction and its influence has also been investigated in earlier works (Mizher et al., 2010; Palhares and Fraga, 2008; Fraga et al., 2009; Palhares and Fraga, 2010). Very recently, Schaefer (Wagner, 2012) worked out the size of 
critical region around CEP in a three flavour (2+1) PQM model where cut off independent renormalization of fermionic vacuum fluctuation has been considered. They calculated critical exponents and higher order non-gaussian moments to identify the fluctuations in particle multiplicities. Since the criticality around CEP is influenced by the presence of strange quark, it is important to have a two flavor calculation in the same model in order to facilitate the comparison with the corresponding size of critical region and nature of criticality obtained in 2+1 flavour QM/PQM model studies.

Here, we will compute the phase diagram in the massless chiral limit and locate the tricritical point (TCP) in the $\mu$ and T plane for the PQMVT and QMVT (QM model with vacuum term) models. Further, we will be investigating the size and extent of critical region around the CEP in phase diagram calculated in the two flavour QM/PQM models with and its without the effect of fermionic vacuum fluctuations. We present the two quark flavour PQM model in the first section. Its subsection gives a brief description of the thermodynamic grand potential, the appropriate renormalization of the fermionic vacuum one loop contribution and the derivation of new model parameters when renormalized vacuum term is added to the effective potential. The next section explores the proximity of QCD tricritical point to the critical end-point and the detail structure of the phase diagram for the QMVT and PQMVT models. The structure of the phase diagram for QM and PQM model and the location of critical end point has also been presented to facilitate the comparison. Its subsection investigates the extent of criticality around CEP where contours of constant baryon number susceptibility ratios and constant scalar susceptibility ratios, have been presented in the $\mu$ and T plane and comparison in all the four models QM, PQM, QMVT and PQMVT, has been made. The critical exponents for the criticality around CEP in all the four models QM, PQM, QMVT and PQMVT have also been computed and discussed. The last section in the end presents summary.

\section{Model}

We will be working in the two flavor quark meson linear sigma model which has been combined with the Polyakov loop potential (Schaefer and Wagner, 2012). In this model, quarks coming in two flavor are coupled to the $S U_{L}(2) \times S U_{R}(2)$ symmetric four mesonic fields $\sigma$ and $\vec{\pi}$ together with spatially constant temporal gauge field represented by Polyakov loop potential. Polyakov loop field $\Phi(\vec{x})$ is defined as the thermal expectation value of color trace of Wilson loop in temporal direction

$$
\begin{array}{r}
\Phi=\frac{1}{N_{c}} \operatorname{Tr}_{c} L, \quad \Phi^{*}=\frac{1}{N_{c}} \operatorname{Tr}_{c} L^{\dagger} \\
\text { and } \quad L(\vec{x})=\mathcal{P} \exp \left[\mathrm{i} \int_{0}^{\beta} \mathrm{d} \tau \mathrm{A}_{0}(\vec{x}, \tau)\right]
\end{array}
$$

where $L(x)$ is a matrix in the fundamental representation of the $S U_{c}(3)$ color gauge group. $\mathcal{P}$ is path ordering, $A_{0}$ is the temporal component of Euclidean vector field and $\beta=T^{-1}$ (Polyakov, 1978). The model 
Lagrangian is written in terms of quarks, mesons, couplings and the Polyakov loop potential $\mathcal{U}\left(\Phi, \Phi^{*}, T\right)$.

$$
\begin{array}{r}
\mathcal{L}_{P Q M}=\mathcal{L}_{Q M}-\mathcal{U}\left(\Phi, \Phi^{*}, T\right) \\
\text { and } \quad \mathcal{L}_{Q M}=\overline{q_{f}}\left[i \gamma^{\mu} D_{\mu}-g\left(\sigma+i \gamma_{5} \vec{\tau} \cdot \vec{\pi}\right)\right] q_{f}+\mathcal{L}_{m}
\end{array}
$$

where $\mathcal{L}_{Q M}$ is the Lagrangian in quark meson linear sigma model. The coupling of quarks with the uniform temporal background gauge field is effected by the following replacement $D_{\mu}=\partial_{\mu}-i A_{\mu}$ and $A_{\mu}=\delta_{\mu 0} A_{0}$ (Polyakov gauge), where $A_{\mu}=g_{s} A_{\mu}^{a} \lambda^{a} / 2 . g_{s}$ is the $S U_{c}(3)$ gauge coupling. $\lambda_{a}$ are Gell-Mann matrices in the color space, a runs from $1 \cdots 8 . q_{f}=(u, d)^{T}$ denotes the quarks coming in two flavors and three colors. Here, $\mathrm{g}$ is the flavor blind Yukawa coupling that couples the two flavor of quarks with four mesons; one scalar $\left(\sigma, J^{P}=0^{+}\right)$and three pseudo scalars $\left(\vec{\pi}, J^{P}=0^{-}\right)$. The mesonic part of the Lagrangian has the following form

$$
\begin{array}{ll}
\qquad \mathcal{L}_{m}=\frac{1}{2}\left(\partial_{\mu} \sigma\right)^{2}+\frac{1}{2}\left(\partial_{\mu} \vec{\pi}\right)^{2}-U(\sigma, \vec{\pi}) \\
\text { and } \quad & U(\sigma, \vec{\pi})=\frac{\lambda}{4}\left(\sigma^{2}+\vec{\pi}^{2}-v^{2}\right)^{2}-h \sigma
\end{array}
$$

$U(\sigma, \vec{\pi})$ is the pure mesonic potential. Here $\lambda$ is quartic coupling of the mesonic fields, $v$ is the vacuum expectation value of scalar field when chiral symmetry is explicitly broken and $h=f_{\pi} m_{\pi}^{2}$.

\section{The Grand Potential, the Renormalized Vacuum Term and Model Parameters}

The thermodynamic grand potential for the PQM model in the mean-field approximation, is written as (Schaefer and Wagner, 2007)

$$
\Omega_{\mathrm{MF}}\left(T, \mu ; \sigma, \Phi, \Phi^{*}\right)=\mathcal{U}\left(T ; \Phi, \Phi^{*}\right)+U(\sigma)+\Omega_{q \bar{q}}\left(T, \mu ; \sigma, \Phi, \Phi^{*}\right) .
$$

The vacuum expectation values $\langle\sigma\rangle=\sigma,\langle\vec{\pi}\rangle=0$. We have used the logarithmic parameterization of the Polyakov loop potential (Rössner et al., 2007; Schaefer and Wagner, 2012). The quark/antiquark contribution in the presence of Polyakov loop reads

$$
\begin{array}{r}
\Omega_{q \bar{q}}\left(T, \mu ; \sigma, \Phi, \Phi^{*}\right)=\Omega_{q \bar{q}}^{\mathrm{vac}}+\Omega_{q \bar{q}}^{\mathrm{T}} \\
=-2 N_{f} \int \frac{d^{3} p}{(2 \pi)^{3}}\left\{N_{c} E_{q} \theta\left(\Lambda^{2}-\vec{p}^{2}\right)+T\left[\ln g_{q}^{+}+\ln g_{q}^{-}\right]\right\}
\end{array}
$$

The first term of the Eq. (6) denotes the fermion vacuum contribution, regularized by the ultraviolet cutoff $\Lambda$. In the second term $g_{q}^{+}$and $g_{q}^{-}$have been defined after taking trace over color space.

$$
\begin{aligned}
& g_{q}^{+}=\left[1+3 \Phi e^{-E_{q}^{+} / T}+3 \Phi^{*} e^{-2 E_{q}^{+} / T}+e^{-3 E_{q}^{+} / T}\right] \\
& \text { and } g_{q}^{-}=\left[1+3 \Phi^{*} e^{-E_{q}^{-} / T}+3 \Phi e^{-2 E_{q}^{-} / T}+e^{-3 E_{q}^{-} / T}\right]
\end{aligned}
$$


Here $\mathrm{E}_{q}^{ \pm}=E_{q} \mp \mu$ and $E_{q}=\sqrt{p^{2}+m_{q}^{2}}$ is the single particle energy of quark/antiquark. The constituent quark mass $m_{q}=g \sigma$ is a function of chiral condensate. In vacuum $\sigma(0,0)=f_{\pi}=93.0 \mathrm{MeV}$. The relevant part of the effective potential in Eq. (5) that will fix the vacuum $(T=0, \mu=0)$ model parameters $\lambda$ and $v$ is the purely $\sigma$ dependent mesonic potential $U(\sigma)$ plus the first term of the Eq. (6) which has been replaced by the renormalized expression $\Omega_{q \bar{q}}^{\mathrm{reg}}$ derived in Ref. (Skokov et al., 2010; Gupta and Tiwari, 2012) for the fermionic vacuum one loop correction.

$$
\begin{aligned}
& \qquad(\sigma)=\Omega_{q \bar{q}}^{\mathrm{reg}}+U(\sigma) \\
& =-\frac{N_{c} N_{f}}{8 \pi^{2}} g^{4} \sigma^{4} \ln \left(\frac{g \sigma}{M}\right)-\frac{\lambda v^{2}}{2} \sigma^{2}+\frac{\lambda}{4} \sigma^{4}-h \sigma \\
& \text { where } \quad \Omega_{q \bar{q}}^{\mathrm{reg}}=-\frac{N_{c} N_{f}}{8 \pi^{2}} m_{q}^{4} \ln \left(\frac{m_{q}}{M}\right) .
\end{aligned}
$$

The first derivative of $\Omega(\sigma)$ with respect to $\sigma$ at $\sigma=f_{\pi}$ in the vacuum is put to zero while the second derivative of $\Omega(\sigma)$ in vacuum gives the mass of $\sigma$

$$
\begin{gathered}
\left.\frac{\partial \Omega_{\mathrm{MF}}\left(0,0 ; \sigma, \Phi, \Phi^{*}\right)}{\partial \sigma}\right|_{\sigma=f_{\pi}}=\left.\frac{\partial \Omega(\sigma)}{\partial \sigma}\right|_{\sigma=f_{\pi}}=0, \\
\text { and } \quad m_{\sigma}^{2}=\left.\frac{\partial^{2} \Omega_{\mathrm{MF}}\left(0,0 ; f_{\pi}, \Phi, \Phi^{*}\right)}{\partial \sigma^{2}}\right|_{\sigma=f_{\pi}}=\left.\frac{\partial^{2} \Omega(\sigma)}{\partial \sigma^{2}}\right|_{\sigma=f_{\pi}}
\end{gathered}
$$

Solving for both the conditions in equation (9), we obtain

$$
\begin{array}{r}
\lambda=\lambda_{s}+\frac{N_{c} N_{f}}{8 \pi^{2}} g^{4}\left[3+4 \ln \left(\frac{g f_{\pi}}{M}\right)\right] \\
\text { and } \lambda v^{2}=\left(\lambda v^{2}\right)_{s}+\frac{N_{c} N_{f}}{4 \pi^{2}} g^{4} f_{\pi}^{2} \\
\text { where } \lambda_{s}=\frac{m_{\sigma}^{2}-m_{\pi}^{2}}{2 f_{\pi}^{2}},\left(\lambda v^{2}\right)_{s}=\frac{m_{\sigma}^{2}-3 m_{\pi}^{2}}{2}
\end{array}
$$

It is evident from the equation (10) that the value of the parameters $\lambda$ and $v^{2}$ have a logarithmic dependence on the arbitrary renormalization scale $M$. However, when we put the value of $\lambda$ and $\lambda v^{2}$ in Eq. (8), the $M$ dependence cancels out neatly after the rearrangement of terms. Finally we obtain

$$
\begin{gathered}
\Omega(\sigma)=-\frac{N_{c} N_{f}}{8 \pi^{2}} g^{4} \sigma^{4} \ln \left(\frac{\sigma}{f_{\pi}}\right)-\frac{\lambda_{r} v_{r}^{2}}{2} \sigma^{2}+\frac{\lambda_{r}}{4} \sigma^{4}-h \sigma, \\
\text { where } \lambda_{r}=\lambda_{s}+\frac{3 N_{c} N_{f}}{8 \pi^{2}} g^{4} \text { and } \lambda_{r} v_{r}^{2}=\left(\lambda v^{2}\right)_{s}+\frac{N_{c} N_{f}}{4 \pi^{2}} g^{4} f_{\pi}^{2}
\end{gathered}
$$

Now the thermodynamic grand potential for the PQM model in the presence of appropriately renormalized fermionic vacuum contribution (PQMVT model) can be written as

$$
\Omega_{\mathrm{MF}}\left(T, \mu ; \sigma, \Phi, \Phi^{*}\right)=\mathcal{U}\left(T ; \Phi, \Phi^{*}\right)+\Omega(\sigma)+\Omega_{q \bar{q}}^{\mathrm{T}}\left(T, \mu ; \sigma, \Phi, \Phi^{*}\right)
$$


One can get the chiral condensate $\sigma$, and the Polyakov loop expectation values $\Phi, \Phi^{*}$ by searching the global minima of the grand potential in Eq. (12) for a given $\mathrm{T}$ and $\mu$

$$
\frac{\partial \Omega_{\mathrm{MF}}}{\partial \sigma}=\frac{\partial \Omega_{\mathrm{MF}}}{\partial \Phi}=\frac{\partial \Omega_{\mathrm{MF}}}{\partial \Phi^{*}}=0
$$

We take $m_{\pi}=138 \mathrm{MeV}, m_{\sigma}=500 \mathrm{MeV}$. The constituent quark mass in vacuum $m_{q}^{0}=310 \mathrm{MeV}$ fixes the Yukawa coupling $g=3.3$.

\section{The Proximity of the TCP to the CEP and The Phase Structure}

The Fig. 1A shows the QMVT and PQMVT model results for real life pion mass, while the Fig. 1B presents the corresponding results for the QM and PQM model. The locations of the TCP in the $\mu$ and $\mathrm{T}$ plane computed for zero pion mass in the QMVT and PQMVT models, have also been shown in Fig. 1A. The TCP does not exist in the phase diagram of QM and PQM models in the chiral limit of zero pion mass because the phase transition, on the temperature axis at $\mu=0$, has been found to be of first order. For calculations with experimental pion mass, solid lines representing the first order chiral phase transition in Fig. 1 merge with the dotted lines (green in color) for the chiral crossover at the CEP (denoted by filled circle). The $\pm 5 \mathrm{MeV}$ error bars (in a range $\mu=100$ to $\mu=160 \mathrm{MeV}$ ) on the dotted line in the upper part of Fig. 1A, signify the ambiguity of pseudo-critical temperature determination for the chiral crossover in the PQMVT model (Gupta and Tiwari 2012) calculations. The thick solid lines around CEP are the contours of constant ratio $\left(R_{q}=2\right)$ of quark number susceptibility obtained in a model calculation to the value of quark number susceptibility for a free quark gas. Since quark number susceptibility diverges at the CEP, such contours signify the extent of critical fluctuations around CEP. The CEP in the QMVT model is located at $\mu_{C E P}=299.35 \mathrm{MeV}$ and $T_{C E P}=32.24 \mathrm{MeV}$ as shown by the filled circle in the lower part of Fig. 1A. It shifts to the higher value on the temperature axis at $T_{C E P}=83.0 \mathrm{MeV}$ and $\mu_{C E P}=295.217 \mathrm{MeV}$ in the PQMVT model due to the influence of Polyakov loop potential.

The filled circle in the lower part of Fig. 1B locates the CEP in QM model at $T_{C E P}=102.09 \mathrm{MeV}$ and $\mu_{C E P}=151.7 \mathrm{MeV}$ and again in the influence of Polyakov loop potential, the CEP in PQM model shifts considerably towards the temperature axis at $T_{C E P}=166.88 \mathrm{MeV}$ and $\mu_{C E P}=81.02 \mathrm{MeV}$ in the upper part of the Fig. 1B. If we compare the location of CEP in QM and PQM models as shown in Fig. 1B to the location of CEP in QMVT and PQMVT models in Fig. 1A, we find a considerably significant shift of CEP to large chemical potential and small temperature values for QMVT and PQMVT models due to the robust influence of fermionic vacuum term inclusion in the effective potential. These results are the extension of our recently reported work (Gupta and Tiwari, 2012) and facilitate the details of model comparison for the two quark flavour case. Further these results are also in qualitative agreement with the recent results of Schaefer et al. (Schaefer and Wagner, 2012) for the $2+1$ flavour case. 

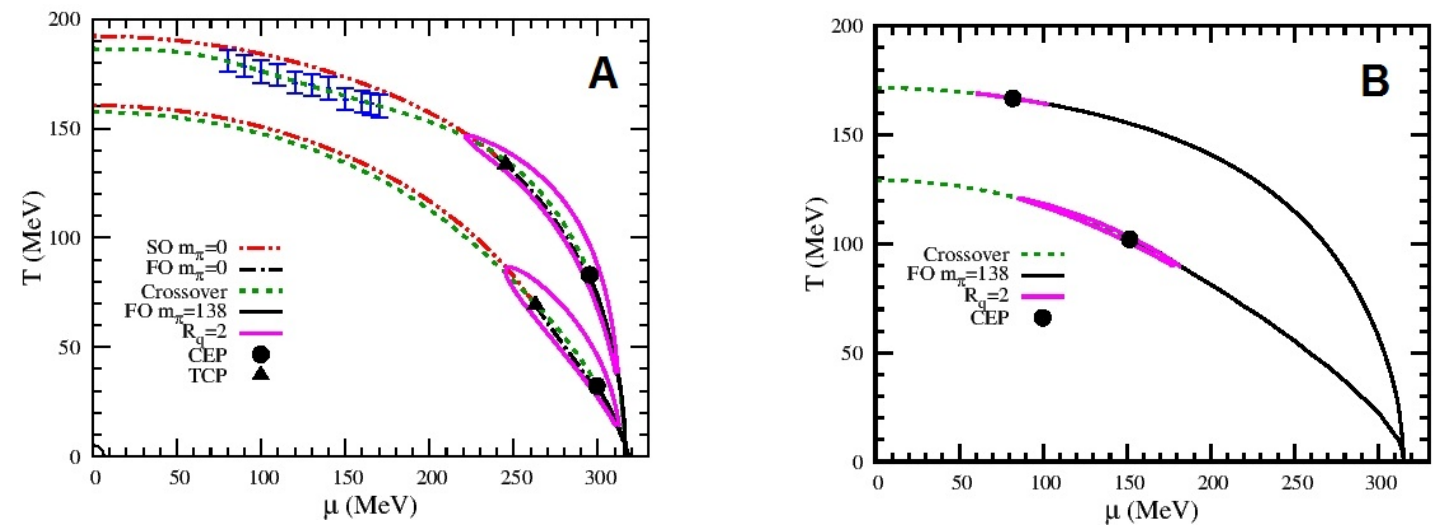

Fig. 1: (A) For calculations with experimental pion mass, solid lines representing the first order chiral phase transition merge with the dotted lines (green in color) for the chiral crossover at the CEP which is denoted by the filled circle. The thick solid lines around CEP are the contours of constant normalized quark number susceptibility $R_{q}=2$. For calculations with zero pion masss, dash lines represent the first order phase transition in the chiral limit of QMVT and PQMVT models while dash dot lines represent the second order transition and the filled triangle is the location of TCP where these two lines merge into each other. Lower part of the Fig. shows the QMVT model results while upper part shows the PQMVT results. (B) Lower part of the Fig. shows the QM model results while upper part shows the PQM results. The line types represent the same thing as in Fig. a for calculations with experimental pion mass

The dash lines of Fig. 1A show the first order phase transition for calculations with zero pion masss in the chiral limit of QMVT and PQMVT models while the dash dot lines represent the second order transition and the filled triangle is the location of TCP where these two lines merge into each other. In the upper part of the Fig. 1A, the filled triangle locates the presence of tricritical point (TCP) at $T_{t}=137.09 \mathrm{MeV}$ and $\mu_{t}=240.14 \mathrm{MeV}$ for PQMVT model calculation. The TCP gets located quite well inside the normalized quark-number susceptibility $\left(R_{q}=2\right)$ contour around the CEP in phase diagram. It means that the shape of the critical region and nature of criticality around CEP, gets influenced by the presence of TCP in the corresponding chiral limit. In a recent NJL/PNJL model calculation by Costa et al., (Costa et al., 2009), the CEP lies closer to the chemical potential axis but the TCP gets located on the periphery of $R_{q}=2$ contour around CEP. In the QMVT model calculation, the tricritical point (TCP) is found at $T_{t}=69.06 \mathrm{MeV}$ and $\mu_{t}=263.0 \mathrm{MeV}$ as denoted by filled triangle in the lower part of the Fig. 1A. Here also the TCP lies quite well inside the $R_{q}=2$ contour on the phase diagram.

\section{Susceptibility Contours, Criticality and Critical Exponents}

The chiral crossover transition is marked by a peak in the quark number susceptibility which becomes sharper and higher as one approaches the CEP in the phase diagram from the crossover side and finally the 
peak diverges at CEP. Enhanced scalar and quark number susceptibilities signify fluctuations near the CEP. Hence we take the contours of normalized constant quark number susceptibilities and scalar susceptibilities, as the measure of criticality around CEP. The ratio of quark-number susceptibility $\chi_{q}$ normalized to the free susceptibility $\chi_{q}^{\text {free }}$ is written as:

$$
\begin{gathered}
R_{q}=\frac{\chi_{q}}{\chi_{q}^{\text {free }}} \quad \text { and } \chi_{q}=-\frac{\partial^{2} \Omega_{\mathrm{MF}}}{\partial \mu^{2}} \\
\text { where } \lim _{m_{q} \rightarrow 0} \chi_{q}(T, \mu)=\frac{\nu_{q}}{6}\left[T^{2}+\frac{3 \mu^{2}}{\pi^{2}}\right] \equiv \chi_{q}^{\text {free }} \\
\text { and } \nu_{q}=2 N_{c} N_{f}=12
\end{gathered}
$$

The first and second partial derivatives of $\sigma, \Phi$ and $\Phi^{*}$ fields with respect to chemical potential contribute in the double derivatives of $\Omega(\sigma), \mathcal{U}_{\log }$ and $\Omega_{\mathrm{q} \overline{\mathrm{q}}}^{\mathrm{T}}$ with respect to chemical potential as given in the appendix A of Ref. (Gupta and Tiwari, 2012).
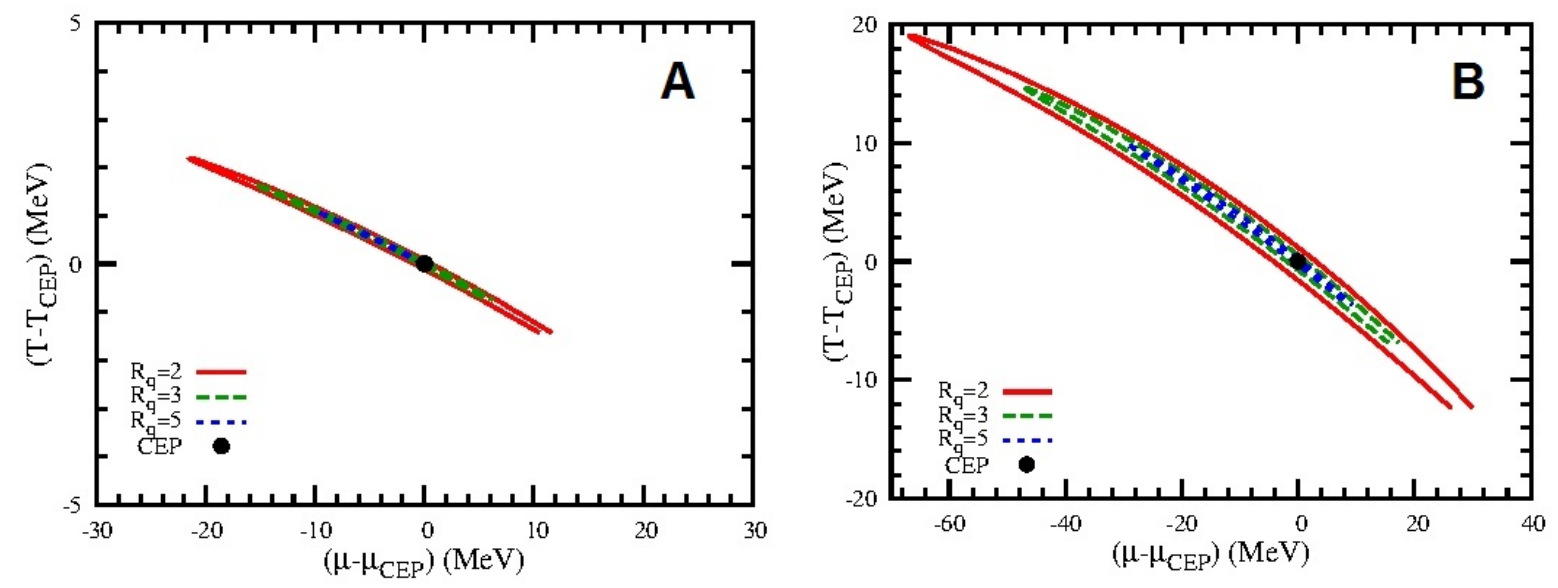

Fig. 2: (A) The contours of three different values for the constant ratios $R_{q}=2,3$ and 5 of quark number susceptibility to the quark susceptibility for the free quark gas, are plotted in the PQM model calculations. (B) Similar contours plotted in the QM model calculations

Contour plots of Fig. 2 in the $\mu, T$ plane around the CEP show three different values of the ratio $R_{q}=$ 2,3 and 5. Comparing the PQM model contours in Fig. 2A) with the QM model contours of Fig. 2B, we conclude that the presence of Polyakov loop potential, compresses the critical region particularly in the T direction similar to findings of Schaefer et al. (Schaefer and Wagner, 2012) in their three flavour calculation. The compression of critical region in the $\mathrm{T}$ direction is much more pronounced in our two quark flavour calculation as can be seen in the spread of $R_{q}=2$ contour on the temperature axis only in a 
small range of $\pm 2.5 \mathrm{MeV}$ near the $T_{C E P}$. The modification in the $\mu$-direction is quite moderate compared to the effect in the T direction. Since the Polyakov loop potential makes chiral crossover faster and sharper, the critical region in the $\mathrm{T}$ direction gets significantly compressed.
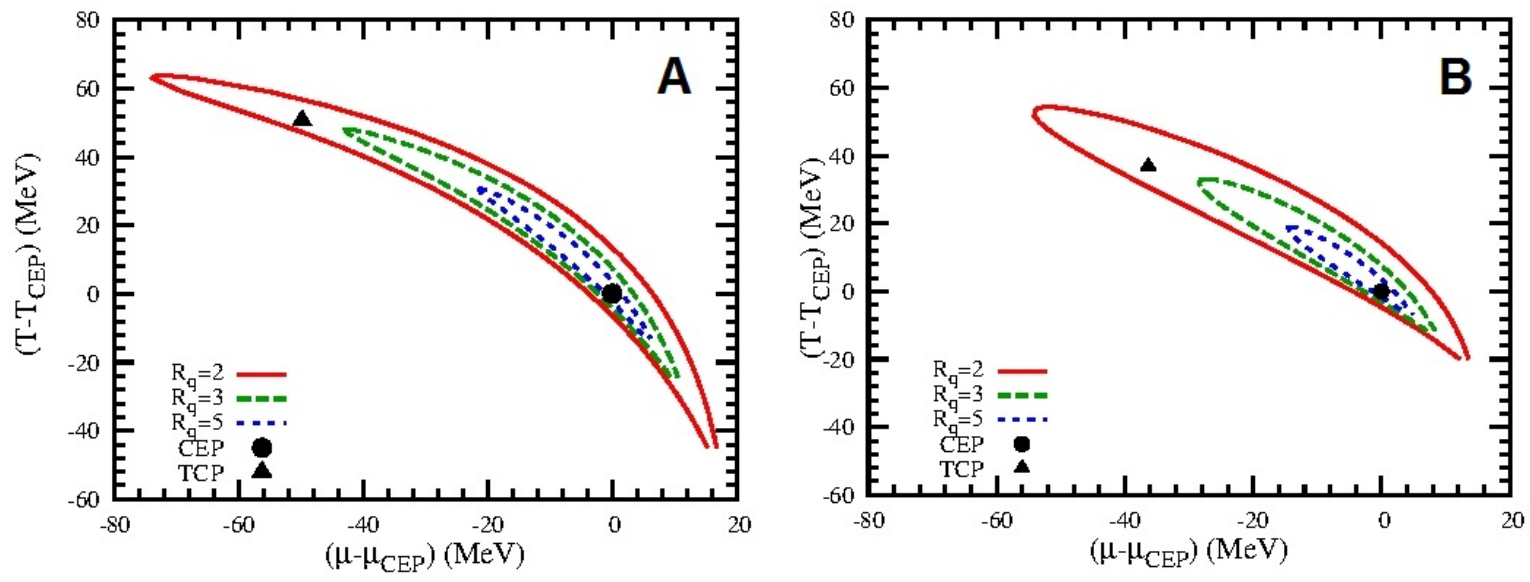

Fig. 3: (A) The contours of three different values for the constant ratios $R_{q}=2,3$ and 5 of quark number susceptibility to the quark susceptibility for the free quark gas,are plotted in the PQMVT model calculations. (B) Similar contours as plotted in the QMVT model calculations

The size of critical region in Fig. 3 is increased in a direction perpendicular to the extended first-order transition line due to the effect of the fermionic vacuum correction. The phase transitions become much smoother and in general get washed out in the influence of fluctuations. This effect is less pronounced in Fig. 3A because the Polyakov loop potential gives rise to a compressed width of critical region in the PQMVT model while the QMVT model results of Fig. 3B show a robust increase in the width of the critical region when Polyakov loop effect is absent. However, the extent and size of critical region in the PQMVT model in Fig. 3A is noticeably larger in both the directions $\mu$ as well as T compared to that of QMVT model results of Fig. 3B. Fermionic vacuum correction shifts CEP to larger chemical potentials in QMVT/PQMVT models.

The zero-momentum projection of the scalar propagator, encodes all fluctuations of the order parameter and it corresponds to the scalar susceptibility $\chi_{\sigma}$. The relation of scalar susceptibility to the sigma mass via $\chi_{\sigma} \sim m_{\sigma}^{-2}$ can be easily verified. We write the scalar susceptibility (Hatta and Ikeda, 2003; Fujii, 2003; Fujii and Ohtani, 2004; Schaefer and Wambach, 2007) and normalized scalar susceptibility $R_{s}(T, \mu)$ as:

$$
\chi_{\sigma}=-\frac{\partial^{2} \Omega_{\mathrm{MF}}}{\partial h^{2}} \quad \text { and } \quad R_{s}(T, \mu)=\frac{\chi_{\sigma}(T, \mu)}{\chi_{\sigma}(0,0)}
$$



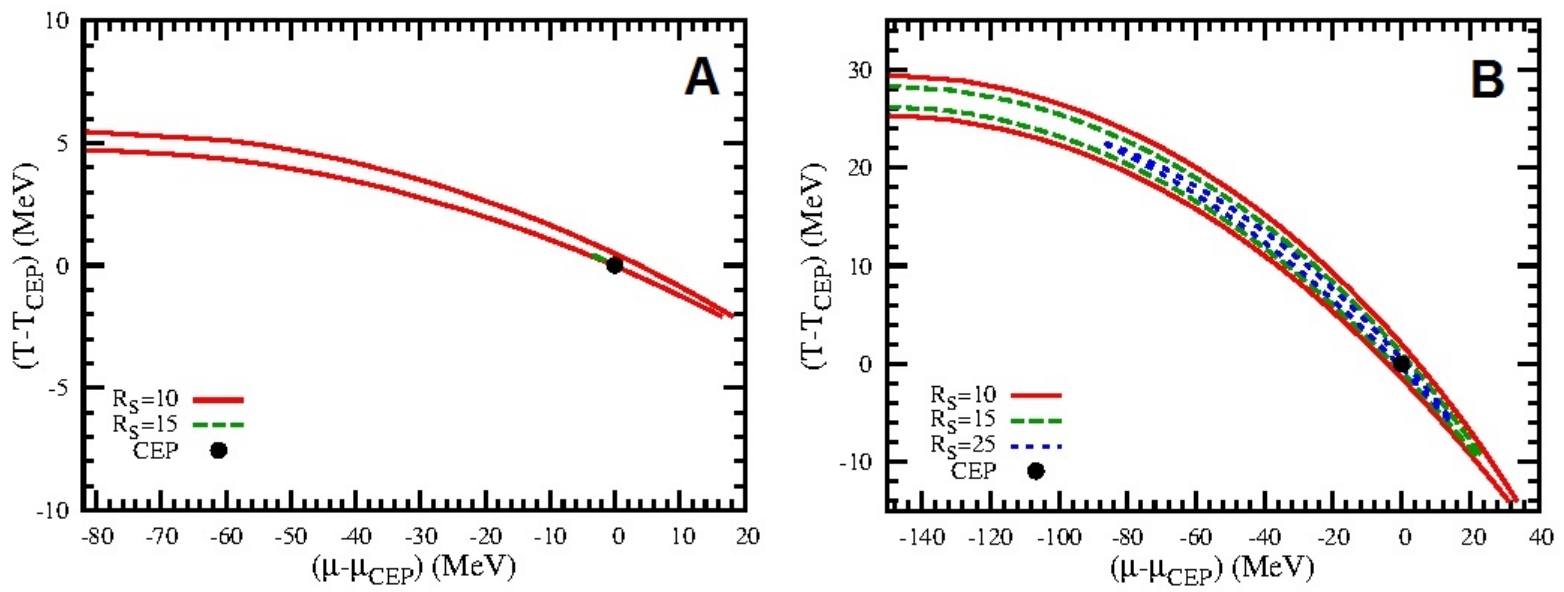

Fig. 4: (A) The contours of different values for the constant ratios $R_{S}=10$ and 15 of $\mathbf{T}$ and $\mu$ dependent scalar susceptibility to the scalar susceptibility at $\mathrm{T}=0$ and $\mu=0 \mathrm{MeV}$, are plotted in the PQM model calculations. (B) Similar contours for the constant ratios $R_{S}=10,15$ and 25 are plotted in the QM model calculations
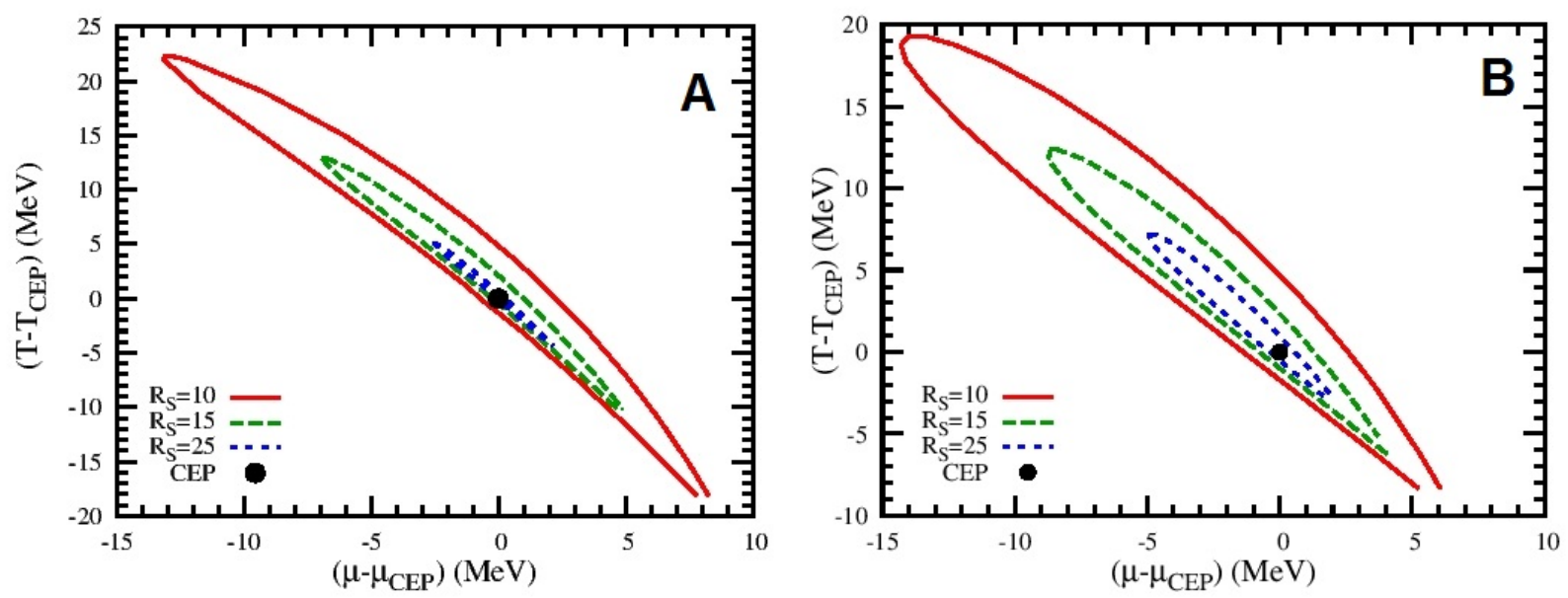

Fig. 5: (A) The contours of three different values for the constant ratios $R_{S}=10,15$ and 25 of $\mathbf{T}$ and $\mu$ dependent scalar susceptibility to the scalar susceptibility at $\mathrm{T}=0$ and $\mu=0 \mathrm{MeV}$,are plotted in the PQMVT model calculations. (B) Similar contours for the constant ratios $R_{S}=10,15$ and 25 are plotted in the QMVT model calculations

The $R_{s}=10$ contour in Fig. 4A is significantly compressed in the $\mathrm{T}$ direction and its extension in the $\mu$ direction is also reduced in comparison to the QM model contours of Fig. 4B. This happens because the temperature or chemical potential variation of $\sigma$ meson mass $m_{\sigma}$ becomes quite fast and rapid on account 
of faster and sharper change of chiral order parameter in the presence of Polyakov loop potential. We do not find contour for $R_{s}=25$ in Fig. 4A because the minimum value of $\sigma$ meson mass does not fall below $100 \mathrm{MeV}$, though the value of $m_{\sigma}$ falls very rapidly and sharply from $500 \mathrm{MeV}$ to $128 \mathrm{MeV}$ giving rise to a very thin and small contour region even for $R_{s}=15$. We get all the contours for $R_{s}=10,15$ and 25 with well defined size in Fig. 4B because the $m_{\sigma}$ variation in QM model is smoother and slower and the minimum of the $m_{\sigma}$ variation approaches almost zero value. We obtain quite well defined and closed contour regions for $R_{s}=10,15$ and 25 in Fig. 5 which again become broader in the direction perpendicular to the extended first order line due to the fermionic vacuum correction in the QMVT and PQMVT model calculations. The fermionic vacuum fluctuations, make the chiral crossover transition very smooth while the Polyakov loop potential makes it sharper and faster and these opposite effects give a typical shape to the quark number susceptibility contours in Fig. 5A in the PQMVT model. Similar effects can be seen in the scalar susceptibility contours also in Fig. 5A. In the influence of fermionic vacuum fluctuations only, the $\chi_{\sigma}$ contours in Fig. 5B in the pure QMVT model are broader and rounded. Next, we will give a brief account of the critical exponents computed from the diverging susceptibilities.

The divergence of quark number susceptibility near the CEP is governed by a power law and the corresponding critical exponents depend on the route through which the singularity (CEP) is approached in the $\mu$ and T plane (Griffiths and Wheeler, 1970). This path dependence decides the shape of the critical region. The calculation of the critical exponents, has been done with the following linear logarithmic fit formula:

$$
\log \chi_{q}=-m \log \left|\mu-\mu_{C E P}\right|+c,
$$

The slope $m$ gives the critical exponent $\epsilon$ and the Y axis intercept $c$ is independent of $\mu$. We have computed the critical exponents for the $\chi_{q}$ in the QM,PQM,QMVT and PQMVT models and the values are given in Table 1.

In Fig. 6A when the $\mu_{C E P}$ is approached from the lower $\mu$ side in the PQMVT model, we obtain a larger critical exponent $\epsilon=m=0.725 \pm 0.00002$ in comparison to the corresponding PQM model result. This larger critical exponent may be the consequence of the modification of criticality around CEP due to the fermionic vacuum fluctuation because we find the presence of TCP well within the $R_{q}=2$ contour near the CEP in the phase diagram of Fig. 1A. If we approach the CEP from the higher $\mu$ side, we find a smaller critical exponent $\epsilon=m=0.6886 \pm 0.0004$ in the result of Fig. 6B. It is pointed out that these exponents calculated in the presence of fermionic vacuum term in the PQM/QM model are different from the meanfield prediction $\epsilon=2 / 3$. The details of comaprison and discussion regarding the critical exponents are given in Ref. (Tiwari, 2012). 


\begin{tabular}{|c|c|c|}
\hline Model & $\mu-\mu_{C E P}<0$ & $\mu-\mu_{C E P}>0$ \\
\hline \hline QM & $0.6379 \pm 0.0002$ & $0.6648 \pm 0.0001$ \\
PQM & $0.6309 \pm 0.0001$ & $0.6668 \pm 0.0001$ \\
QMVT & $0.720 \pm 0.00005$ & $0.6938 \pm 0.0002$ \\
PQMVT & $0.725 \pm 0.0002$ & $0.6886 \pm 0.0004$ \\
\hline
\end{tabular}

Table 1: Critical exponents of the quark-number susceptibility in the QM, PQM, QMVT and PQMVT models for two different paths parallel to the chemical potential axis approaching the $\mu_{C E P}$ from the lower $\mu<\mu_{C E P}$ and higher $\mu>\mu_{C E P}$ side
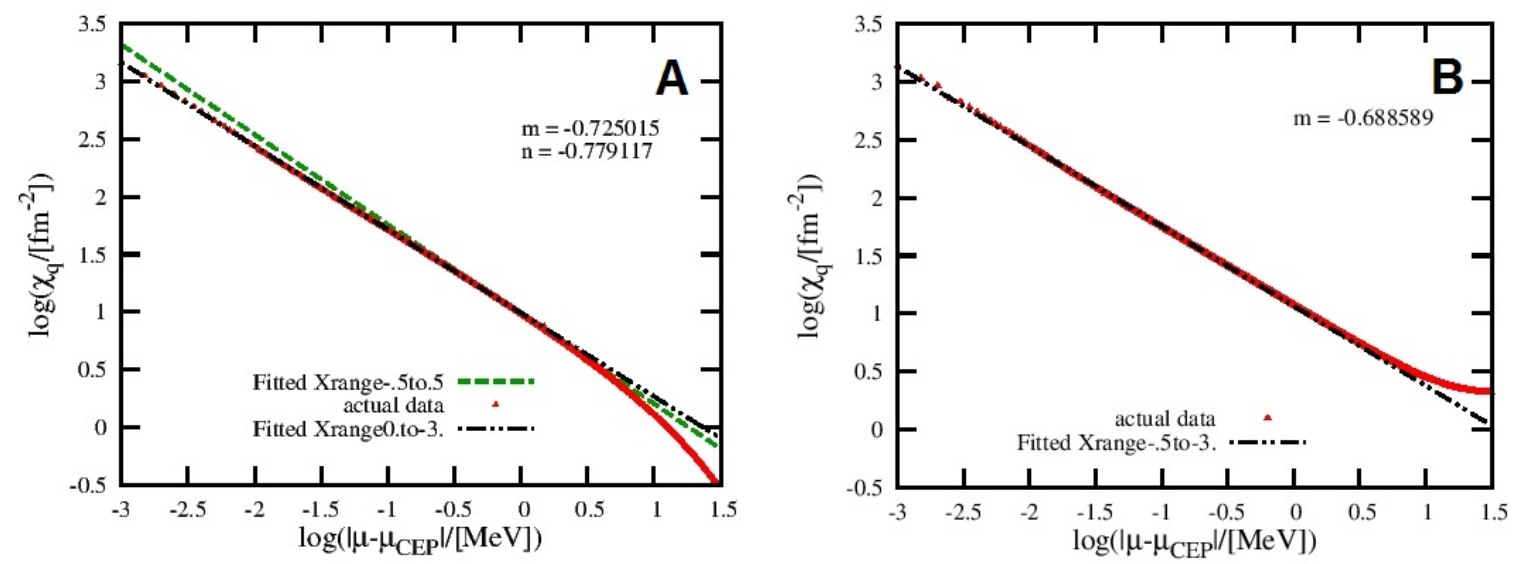

Fig. 6: (A) Plot of the logarithm of $\chi_{q}$ as a function of the logarithm of $\mu-\mu_{C E P}$ close to the CEP in PQMVT model when the $\mu_{C E P}$ is approached from the lower $\mu$ side (B) Same plot as in Fig. a in PQMVT model when the $\mu_{C E P}$ is approached from the higher $\mu$ side

\section{Summary and Conclusion}

The chiral transition on the temperature axis at zero chemical potential becomes significantly smoother due to the fermionic vacuum correction and we find a considerably significant shift of the CEP to large chemical potential and small temperature values for QMVT and PQMVT model computations. In contrast Polyakov loop potential leads to quite a sharp transition and we get a very small and narrow critical region near the CEP in PQM model. Critical region near the CEP for the PQMVT model is significantly large and it is stretched in the direction parallel to the crossover line. The width of the critical region increases in perpendicular direction to the extended first order line due to the fermionic vacuum fluctuation. We find tricritical point (TCP) in the phase diagram of PQMVT/QMVT model for calculations in the chiral limit of 
$m_{\pi}=0$. The proximity of TCP to the CEP has been quantified by plotting the constant normalized quarknumber susceptibility $\left(R_{q}=2\right)$ contours around CEP in the phase diagrams of PQMVT and QMVT models. The second cumulant of the net quark number fluctuations on these contours is double to that of the free quark gas value and such enhancements are the signatures of CEP for the heavy-ion collision experiments. The TCP location is quite well inside the $R_{q}=2$ contour on the phase diagram of both the models QMVT as well as PQMVT. Hence TCP will influence the critical behavior near CEP.

\section{Acknowledgments}

I am extremely thankful to Prof. Sanjay K Ghosh and all other organizers of the International Conference on Matter Under Extreme Conditions organized during 15 to 17 January 2014 at J.C. Bose Institute Kolkota for providing me a wonderful hospitality. Suggestions and computational helps by Rajarshi Ray are specially acknowledged.

\section{References}

1. Adams J et al., (STAR Collaboration) (2005) Experimental and theoretical challenges in the search for the quark gluon plasma: The STAR Collaboration's critical assessment of the evidence from RHIC collisions $\mathrm{Nucl}$ Phys A757 102

2. Aggarwal MM et al., (2010) Higher Moments of Net-proton Multiplicity Distributions at RHIC Phys Rev Lett 105022302

3. Allton CR, Ejiri S, Hands SJ, Kaczmarek O, Karsch F, Laermann E and Schmidt C (2003) The Equation of state for two flavor QCD at nonzero chemical potential Phys Rev D68 (2003) 014507

4. Allton CR, Doring M, Ejiri S, Hands SJ, Kaczmarek O, Karsch F, Laermann E and Redlich K (2005) Thermodynamics of two flavor QCD to sixth order in quark chemical potential Phys Rev D71 054508

5. Aoki Y, Androdi G, Fodor Z, Katz SD and Szabo KK (2006) The Order of the quantum chromodynamics transition predicted by the standard model of particle physics Nature $\mathbf{4 4 3} 675$

6. Aoki Y, Fodor Z, Katz SD and Szabo KK (2006) The QCD transition temperature: Results with physical masses in the continuum limit Phys Lett B643 (2006) 46

7. Aoki Y et al., (2009) The QCD transition temperature: results with physical masses in the continuum limit II JHEP 06088

8. Asakawa M and Yazaki K (1989) Chiral Restoration at Finite Density and Temperature Nucl Phys A504 668

9. Barducci A, Casalbuoni R, De Curtis S, Gatto R and Pettini G (1989) Chiral Symmetry Breaking in QCD at Finite Temperature and Density Phys Lett B231 463

10. Barducci A, Casalbuoni R, De Curtis S, Gatto R and Pettini G (1990) Chiral Phase Transitions in QCD for Finite Temperature and Density Phys Rev D41 1610

11. Barducci A, Casalbuoni R, Pettini G and Gatto R (1994) Chiral phases of QCD at finite density and temperature Phys Rev D49 426 
12. Bazavov A et al., (2012) The chiral and deconfinement aspects of the QCD transition Phys Rev D85 054503

13. Berges J and Rajagopal K (1999) Color superconductivity and chiral symmetry restoration at nonzero baryon density and temperature Nucl Phys B538 215

14. Borsanyi S et al., (2010) Is there still any $T_{c}$ mystery in lattice QCD? Results with physical masses in the continuum limit III JHEP 09073

15. Brouzakis N and Tetradis N (2004) The phase structure of the Polyakov-quark-meson model beyond mean field Nucl Phys A742 144

16. Costa P, de Sousa CA, Ruivo MC and Hansen H (2009) The QCD critical end point in the PNJL model Europhys Lett 8631001

17. Ejiri S, Karsch F and Redlich K (2006) Hadronic fluctuations at the QCD phase transition Phys Lett $\mathbf{B 6 3 3} 275$

18. Fodor $\mathrm{Z}$ and Katz SD (2002) A New method to study lattice QCD at finite temperature and chemical potential Phys Lett $\mathbf{B 5 3 4} 87$

19. Fodor Z, Katz SD and Szabo KK (2003) The QCD equation of state at nonzero densities: Lattice result Phys Lett $\mathbf{B 5 6 8} 73$

20. Forcrand P de and Philipsen O (2002) The QCD phase diagram for small densities from imaginary chemical potential Nucl Phys B642 290

21. Fraga ES, Palhares LF and Pinto MB (2009) Nonperturbative Yukawa theory at finite density and temperature Phys Rev D79 065026

22. Fujii H (2003) Scalar density fluctuation at critical end point in NJL model Phys Rev D67 094018

23. H Fujii and M Ohtani (2004) Sigma and hydrodynamic modes along the critical line Phys Rev D70 014016

24. Fukushima K (2010) Effective Model Approach to the Dense State of QCD Matter arXiv:1008.4322

25. Griffiths RB and Wheeler J (1970) Critical Points in Multicomponent Systems Phys Rev A2 1047

26. Gupta S, Luo X, Mohanty B, Ritter HG and Xu N (2011) Scale for the Phase Diagram of Quantum Chromodynamics Science 3321525

27. Gupta US and Tiwari VK (2010) Meson Masses and Mixing Angles in 2+1 Flavor Polyakov Quark Meson Sigma Model and Symmetry Restoration Effects Phys Rev D81 054019

28. Gupta US and Tiwari VK (2012) Revisiting the Phase Structure of the Polyakov-quark-meson Model in the presence of Vacuum Fermion Fluctuation Phys Rev D85 014010

29. Halasz MA, Jackson AD, Shrock RE, Stephanov MA and Verbaarschot JJM (1998) On the phase diagram of QCD Phys Rev D58 096007

30. Harada M and Shibata A (1999) Chiral phase transition of QCD at finite temperature and density from SchwingerDyson equation Phys Rev D59 014010

31. Hatta Y and Ikeda T (2003) Universality, the QCD critical / tricritical point and the quark number susceptibility Phys Rev D67 014028

32. Herbst TK, Pawlowski JM and Schaefer BJ (2011) The phase structure of the Polyakov-quark-meson model beyond mean field Phys Lett B696 58

33. Jeon S and Koch V (2000) Charged particle ratio fluctuation as a signal for QGP Phys Rev Lett 852076 
34. Karsch F (2002) Lattice QCD at high temperature and density Lect Notes Phys $\mathbf{5 8 3} 209$

35. Koch V (2008) Hadronic Fluctuations and Correlations arXiv:0810.2520

36. Layek B, Mishra AP, Srivastava AM and Tiwari VK (2006) Baryon inhomogeneity generation in the quarkgluon plasma phase. Phys Rev D73 103514

37. Luo X, Mohanty B, Ritter HG and Xu N (2012) Search for the QCD Critical Point: Higher Moments of Netproton Multiplicity Distributions Physics of Atomic Nuclei 75676

38. Mao H, Jin J and Huang M (2010) Phase diagram and thermodynamics of the Polyakov linear sigma model with three quark flavors $J$ Phys $\mathbf{G 3 7} 035001$

39. Marko G and Szep ZS (2010) Influence of the Polyakov loop on the chiral phase transition in the two flavor chiral quark model Phys Rev D82 065021

40. Meyer-Ortmanns H (1996) Phase transitions in quantum chromodynamics Rev Mod Phys 68473

41. Mizher AJ, Chernodub MN and Fraga ES (2010) Phase diagram of hot QCD in an external magnetic field: possible splitting of deconfinement and chiral transitions Phys Rev D82 105016

42. Mohanty B (2009) QCD Phase Diagram: Phase Transition, Critical Point and Fluctuations Nucl Phys A830 (2009) 899c

43. Muller B (1995) Physics and signatures of the quark - gluon plasma Rep Prog Phys 58611

44. Nonaka C and Asakawa M (2005) Hydrodynamical evolution near the QCD critical end point Phys Rev C71 044904

45. Palhares LF and Fraga ES (2008) Perturbative Yukawa theory at finite density: the role of masses and renormalization group flow at two loops Phys Rev D78 025013

46. Palhares LF and Fraga ES (2010) Droplets in the cold and dense linear sigma model with quarks Phys Rev D82 125018

47. Philipsen O (2011) Status of the QCD phase diagram from lattice calculations arXiv:1111.5370

48. Pisarski RD (2000) Quark-gluon plasma as a condensate of Z(3) Wilson lines Phys Rev D62 111501(R)

49. Pisarski RD and Wilczek F (1984) Vacuum fluctuations and the thermodynamics of chiral models Phys Rev D29 338

50. Polyakov AM (1978) Thermal properties of gauge fields and quark liberation Phys Lett B72 477

51. Rischke DH (2004) The quark-gluon plasma in equilibrium Prog Part Nucl Phys 52197

52. Rößner S, Ratti C and Weise W (2007) Polyakov loop, diquarks, and the two-flavor phase diagram Phys Rev D75 034007

53. Schaefer BJ and Wagner M (2012) Susceptibilities near the QCD (tri)critical point Phys Rev D85 034027

54. Schaefer BJ and Wambach J (2007) Susceptibilities near the QCD (tri)critical point Phys Rev D75 085015

55. Schaefer BJ, Pawlowski JM and Wambach J (2007) Phase Structure of the Polyakov-quark meson model Phys Rev D76 074023

56. Schaefer BJ and Wagner M (2009) On the QCD phase structure from effective models Prog Part Nucl Phys 62 381

57. Schaefer BJ, Wagner M and Wambach J (2009) QCD thermodynamics with effective models PoS (CPOD 2009) 
017

58. Schaefer BJ, Wagner M and Wambach J (2010) Thermodynamics of (2+1) flavor QCD: Confronting Models with Lattice Studies Phys Rev D81 074013

59. Skokov V, Friman B, Nakano E, Redlich K and Schaefer BJ (2010) Vacuum fluctuations and the thermodynamics of chiral models Phys Rev D82 034029

60. Son DT and Stephanov MA (2004) Dynamic universality class of the QCD critical point Phys Rev D70 056001

61. Stephanov MA, Rajagopal K and Shuryak EV (1999) Event-by-event fluctuations in heavy ion collisions and the QCD critical point Phys Rev D60 114028

62. Stephanov MA, Rajagopal K and Shuryak EV (1998) Signatures of the tricritical point in QCD Phys Rev Lett 814816

63. Svetitsky B (1986) Symmetry Aspects of Finite Temperature Confinement Transitions Phys Rep 1321

64. Tiwari VK (2012) Exploring criticality in the QCD-like two quark flavour models Phys Rev D86 094032. 